\title{
A Wireless Power Transfer System for Electrical Vehicles Exploiting Magnetically Coupled Resonance and a Tapped Capacitance Impedance Transformer
}

\author{
Mihai D. Rotaru \\ University of Southampton, UK \\ mr@ecs.soton.ac.uk
}

\author{
Tan Yen Kheng \\ Energy Research Institute @NTU, \\ Singapore \\ TANYK@ntu.edu.sg
}

\author{
Jan K. Sykulski \\ University of Southampton, UK \\ jks@soton.ac.uk
}

\begin{abstract}
The input impedance of a wireless power transfer system is heavily influenced by variation of the load impedance, distance between coils and presence of any massive conducting or shielding structures that may exist around the system. To achieve the maximum efficiency for the wireless link transfer one has to ensure good matching between the power source and the wireless power transfer (WPT) system for different working conditions. In this paper an equivalent circuit of the WPT which accounts for the effects of the proximity of massive conducting structures is developed. A novel tapped capacitance impedance transformer for dynamic matching purposes is introduced and its presence incorporated into the equivalent circuit; its effect on the efficiency of the system is investigated.
\end{abstract}

Index Terms-- Equivalent circuit model, matching circuit, resonant coupled coils, non-radiative power transfer, tapped capacitor network, wireless power transfer (WPT).

\section{INTRODUCTION}

In recent years the automotive industry has seen a transformation driven by the need to reduce the $\mathrm{CO}_{2}$ emissions. Hybrid electric and full electric vehicles begin to penetrate the consumer market; however, the fact that such vehicles depend on battery packs of limited storage capabilities, and need to be charged in reasonable time, still restricts the full acceptance of such vehicles. Recharging the vehicle batteries using a cable power connection, although relatively efficient, is inconvenient; hence the concept of wireless power transfer was suggested [1,2].

One of the methods proposed for wireless power transfer for electric vehicle applications is using the principle of coupled magnetic resonances, first introduced in 2007 by Soljacic et al [3]. Although in principle the magnetic coupled resonance technique is a very efficient method to transfer power over a medium distance at frequencies spanning from $\mathrm{kHz}, \mathrm{MHz}$ up to $\mathrm{GHz}$, its efficiency is strongly influenced not only by the distance between the two coupled coils but also by variations in the load impedance as well as by any massive conducting structures existing in the vicinity of the system. All these effects are very important in the design of a realistic WPT system for electric vehicle applications.

There have been extensive work and studies on the design and optimization of magnetically coupled (inductive) power links over the past several years [3]-[10]. Different approaches to model and analyse the problem have been proposed; a coupled-mode theory (CMT) and an equivalent circuit model are some of the most popular approaches $[5,6]$. Several circuit topologies for different applications have been suggested, analysed and some realized [8, 9]. It has been shown that - depending on the distance and power range compensation topologies such as series-series, series-parallel or parallel-parallel will result in different performance. For the application studied in this work, which requires large amount of power to be wirelessly transferred to the electrical vehicle being charged, the most suitable compensation topology was found to be the series-series type [9].

In our previous work [10] we have demonstrated, through an equivalent circuit extracted from a 3D FEM model, that by changing the load resistance the efficiency of the system could be improved, even for a large separation between the two magnetically coupled resonators; however, the maximum efficiency point may not correspond to the maximum power that can be handled by the system. In this paper the equivalent circuit model is developed further to include the effects of any massive conducting structures (or shielding); moreover, the impedance matching necessary to achieve efficient wireless power transfer is accomplished by using a tapped capacitance impedance transformer rather than through a change in the load impedance.

\section{EQUIVALENT CIRCUIT MODELLING}

The geometry and the structure of the WPT system under consideration has been described in detail in our previous publication [10] and therefore will not be repeated here. The system is designed to work at a frequency of $96.5 \mathrm{kHz}$. The resonant circuit of the system consists of a wound coil of $240.7 \mu \mathrm{H}$ and two series capacitors of $22.6 \mathrm{nF}$ each.

The equivalent circuit through which the two magnetically coupled resonant coils can be modelled is presented in Fig. 1. The inductances $(L 1, L 2)$ and capacitances $(C 1, C 2, C 3$ and $C 4)$ model the resonant nature of the coils, while the resistors $(R 1, R 2)$ represent losses in the system. Both the radiation and resistive losses are modelled by the two resistors. The two coils are coupled through their mutual inductance (L12) as shown in the equivalent circuit representation. The equivalent circuit shown in Fig. 1 was extracted from a full wave 3D 
simulation and has been shown to model well the electromagnetic problem [10].

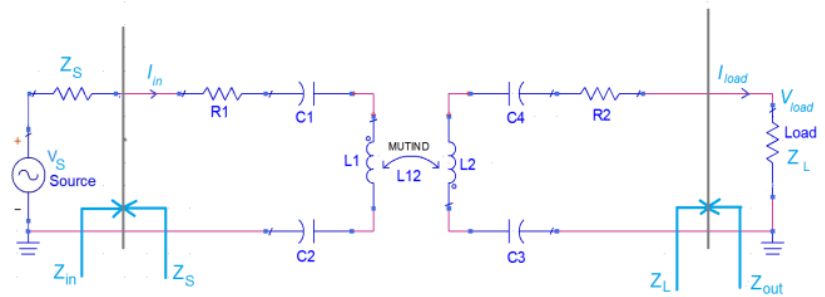

Fig. 1. Equivalent circuit model for two magnetically coupled resonant coils. [10]

From the above circuit the input and output impedances can be written as:

$$
\begin{gathered}
Z_{\text {in }}(\omega)=\left(j \omega L_{1}+1 /\left(j \omega C_{1}\right)+1 /\left(j \omega C_{2}\right)+R_{1}\right)+ \\
\left(\omega^{2} \cdot L_{12}^{2}\right) /\left(j \omega L_{2}+1 /\left(j \omega C_{3}\right)+1 /\left(j \omega C_{4}\right)+R_{2}+Z_{L}\right)(1) \\
Z_{\text {out }}(\omega)=\left(j \omega L_{2}+1 /\left(j \omega C_{3}\right)+1 /\left(j \omega C_{4}\right)+R_{2}\right)+ \\
\left(\omega^{2} \cdot L_{12}^{2}\right) /\left(j \omega L_{1}+1 /\left(j \omega C_{1}\right)+1 /\left(j \omega C_{2}\right)+R_{1}+Z_{S}\right)(2)
\end{gathered}
$$

At resonance the two impedances $Z_{\text {in }}$ and $Z_{\text {out }}$ become purely real (as shown in Fig. 2 where the real in imaginary parts of $Z_{i n}$ are presented). If the two coils are identical the resonance occurs at $\omega_{0}=1 / \sqrt{L C}$, where $L=L 1=L 2$ and $C=(C 1 \cdot C 2) /(C 1+C 2)=(C 3 \cdot C 4) /(C 3+C 3)$.

Hence they can be re-written in the following form

$$
\begin{aligned}
& Z_{\text {in }}\left(\omega_{0}\right)=\left(R_{1}\right)+\left(\omega_{0}^{2} \cdot L_{12}^{2}\right) /\left(R_{2}+Z_{L}\right) \\
& Z_{\text {out }}\left(\omega_{0}\right)=\left(R_{2}\right)+\left(\omega_{0}^{2} \cdot L_{12}^{2}\right) /\left(R_{1}+Z_{S}\right)
\end{aligned}
$$

As discussed in detail in [10] there are large variations in the input impedance of this system as the distance between the coils is changed and/or the load impedance varies. Furthermore, these variations affect strongly the efficiency and power capability of the WPT system. It is desirable to maximise the efficiency of the wireless power transfer scheme, hence matching the source and load to the input and output impedance, respectively, is necessary. As explained in [10] a highly efficient wireless link can be obtained (even for relatively large distances) if the load impedance is changed while the distance between coils is varied such that the input

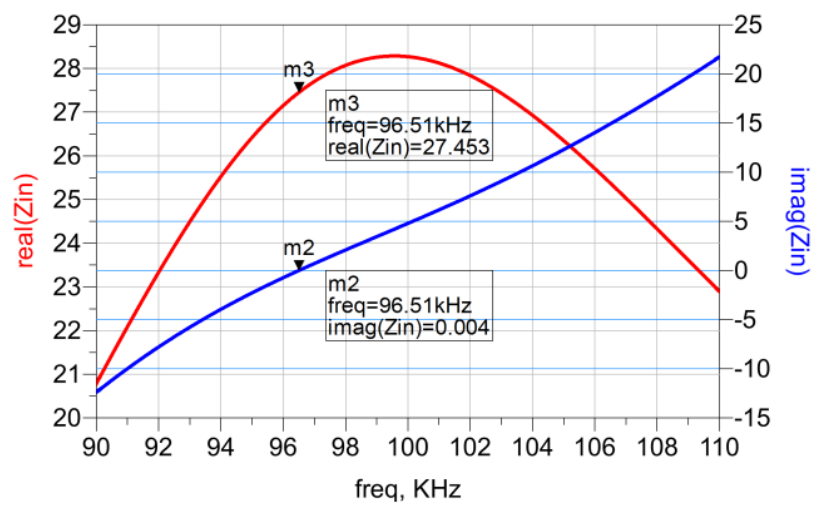

Fig. 2. Real and imaginary components of $Z_{i n}$ when load impedance is $50 \mathrm{ohms}$ and distance between coils is $100 \mathrm{~mm}$. $\omega_{0}$ for the system under study is $\left(96.5 \mathrm{e}^{3}\right) * 2 \pi[\mathrm{rad} / \mathrm{s}]$. impedance matches the source impedance.

The efficiency of this system can be expressed in terms of the equivalent circuit elements as [10]

$$
\eta=\left(Z_{L} \omega_{0}^{2} L_{12}^{2}\right) /\left(\omega_{0}^{2} L_{12}^{2}\left(R_{2}+Z_{L}\right)+R_{1}\left(R_{2}+Z_{L}\right)^{2}\right)
$$

Although the equation above quantifies the efficiency of the wireless transfer process, it does not account for what happens at the interface between the WPT system and the power source. The impedance mismatch between the source impedance $\left(Z_{s}\right)$ and input impedance $\left(Z_{\text {in }}\right)$ has an important role in regulating the amount of power that can be transferred through the WPT system, hence it should be considered in the design and analysis of such systems.

It has been argued in [7] and [10] that a general and complete definition of efficiency of a fully connected WPT system is

$$
\eta_{\text {full }}=\left(4 Z_{s} Z_{L} \omega_{0}^{2} L_{12}^{2}\right) /\left(\omega_{0}^{2} L_{12}^{2}+\left(R_{1}+Z_{s}\right)\left(R_{2}+Z_{L}\right)\right)(6)
$$

The above equation can be re-arranged and written in terms of equation (5) as follows

$$
\eta_{f u l l}=\eta\left(1-\left|\Gamma_{i n}\right|^{2}\right)
$$

where $\eta$ is given by equation (5) and $\Gamma_{\text {in }}$ is the input reflection coefficient defined as

$$
\Gamma_{\text {in }}=\left(Z_{\text {in }}-Z_{s}\right) /\left(Z_{\text {in }}+Z_{s}\right)
$$

It can be noticed from equation (7) that the efficiency of a fully connected and loaded WPT system has two distinctive components: one which is solely related to the two coils and their quality factor, and a second one which is associated with the matching between the source impedance and the input impedance of the WPT system. In this study it is argued that although the reflection coefficient is a very important design parameter for a WPT system it does not affect the efficiency of the system but rather the amount of power that the system will be transferring, and hence minimizing the reflection coefficient and maximizing the quality factor of the magnetically coupled coils will lead to an optimum WPT system.

With the above observations in mind it will be informative to analyse the behaviour of the WPT under test. The load impedance for the system under study is $11.75 \Omega$. This load fits the requirements of the electrical vehicle which has an on board charger with 120-240 V and a maximum current of 13 A. This means that the load could be between 9 and $18 \Omega$; however, from a practical implementation point of view, for lab testing purposes $40 \times 470 \Omega 50 \mathrm{~W}$ parallel resistances were used. The source impedance was considered to be $0.1 \Omega$. Although this value has been chosen for demonstration of the concept only and has not been measured, it is expected that in practical systems the source impedance will be relatively small, hence a value of $0.1 \Omega$ may be considered as relevant 


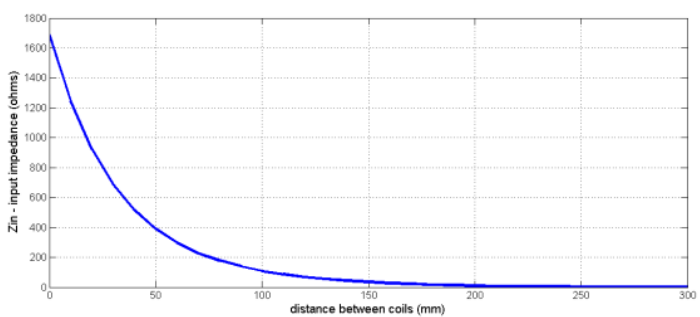

Fig. 3. Magnitude of the input impedance $\left(Z_{i n}\right)$ when the distance between coils is varied (from 0 to $300 \mathrm{~mm}$ ).

to the situation under study. With the above values introduced into the equivalent circuit model the input impedance of the system was calculated for different distances between the two coils. As shown in Fig. 3 the input impedance varies from more than $1000 \Omega$ for distance below $20 \mathrm{~mm}$ to less than $1 \Omega$ for distances above $290 \mathrm{~mm}$.

Two extreme situations were simulated using the equivalent circuit presented in Fig. 2 and the results are discussed in the next paragraphs. For both cases the input voltage has an amplitude of $100 \mathrm{~V}$.

First, the case when the two coils are $20 \mathrm{~mm}$ apart was simulated. In this situation the two coils are tightly coupled; however, the input impedance $Z_{\text {in }}$ is very high at $916 \Omega$ (Fig. 4.a).

In the second extreme case considered the distance between the coils was assumed to be $290 \mathrm{~mm}$. In this case the input impedance of the WPT is only $0.865 \Omega$; however, the two coils are loosely coupled (Fig. 5.a).
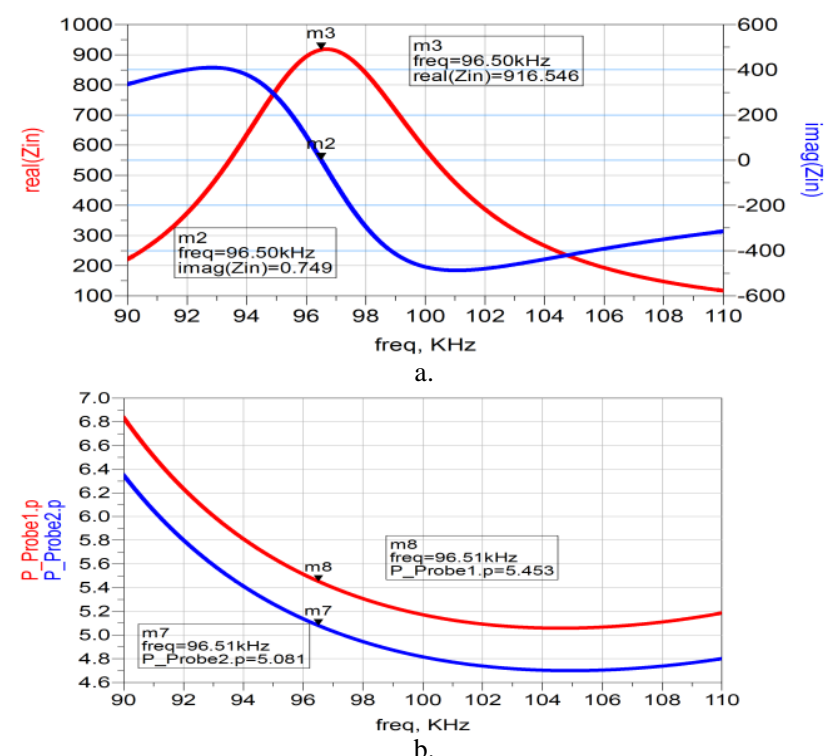

Fig 4. a. Real and imaginary components of $Z_{\text {in }}$ when load impedance is $11.75 \Omega$ and distance between coils is $20 \mathrm{~mm}$. b. Power in and power delivered to the load.

For the first case, although the two coils are very close, the input impedance is very high, hence the input current is small. For the simulated case for a source voltage with an amplitude of $100 \mathrm{~V}$ the input current has an amplitude of only 0.109 A. Hence the input power is only $5.45 \mathrm{~W}$, however the output

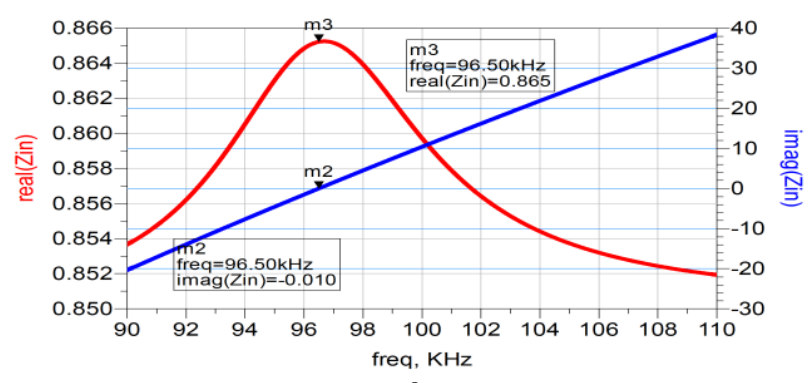

a.

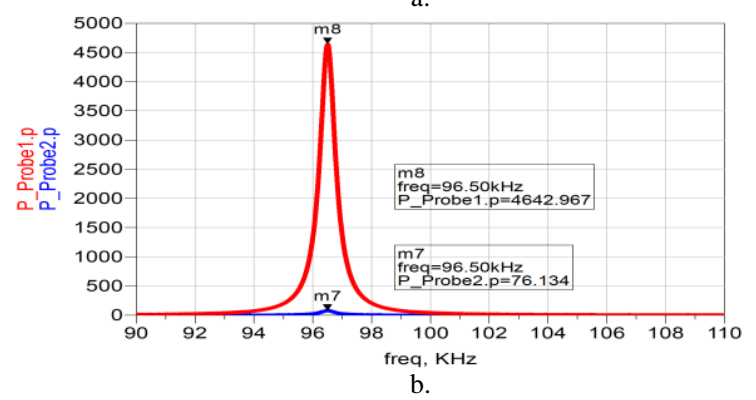

Fig 5. a. Real and imaginary components of $Z_{\text {in }}$ when load impedance is $11.75 \Omega$ and distance between coils is $290 \mathrm{~mm}$. b. Power in and power delivered to the load.

power is $5.08 \mathrm{~W}$ which implies a $93 \%$ efficiency (Fig. 4.b). On the other hand, for the second case, the input impedance is small, therefore the input current will be large; in the simulated cases the amplitude of the primary current was 103.6 A. This large value of current translates to a large input power of $4643 \mathrm{~W}$; however, only $76.15 \mathrm{~W}$ are transferred to the second coil which results in a very poor efficiency of $1.6 \%$ (Fig. 5.b). It has to be mentioned that the unloaded quality factor of the two coupled resonators is about 85.75. If, for example, the quality factor of the resonators could be increased to around 2000, the efficiency in the second case could rise to $30 \%$; however this would mean that the resistance of the coils should be reduced from the $0.85 \Omega$ measured value to $0.035 \Omega$, which may not be practical. The efficiency would be $99.71 \%$ in the first case if such high quality resonators were available. Although high quality factor resonators are desirable it is very difficult to make coils with such a low self-resistance. On the other hand, one can try to match the input impedance of the WPT system to the source impedance, so that when good coupling exists between the two coils more power could be transferred through the system at a higher efficiency. The following section will discuss in detail a possible solution based on a tapped capacitance impedance transformer that could be applied to the WPT systems.

\section{TAPPED CAPACITANCE IMPEDANCE TRANSFORMER}

Tapped capacitance impedance transformer is a method used usually in RF design to match different stages in a RF system [11]. To our knowledge this method has not been applied before to a wireless power transfer system, such as the one discussed here. The idea behind this method can be inferred from Fig. 6. 
The network in Fig. 6 can be used as an impedance transformer. The relationship between the $R_{s}$ and $R_{l}$ has been shown to be [11]

$$
R_{l} \approx R_{S} \cdot\left(1+C_{B} / C_{A}\right)^{2}
$$

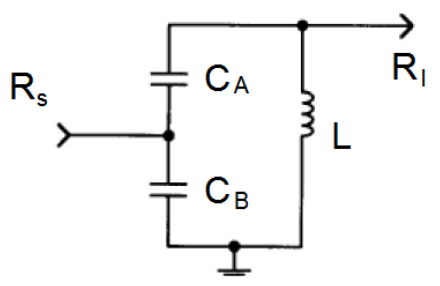

Fig. 6. Tapped capacitance impedance transformer

The role of the inductor $L$ is to resonate the series capacitance $\left(C_{A} C_{B}\right) /\left(C_{A}+C_{B}\right)$ at frequency of interest. Although impedance transformers could be designed using resistance networks, it is expected that the losses would be higher in such a network, thus reducing the efficiency of a WPT system, they were therefore avoided in this study. Another advantage of this approach is that it is much easier to build this network than a conventional transformer.

To demonstrate the effectiveness of this proposed approach a model of the WPT system with a tapped capacitance network inserted between the source and the primary coils has been simulated. However, designing the network in Figure 4 to work in conjunction with the WPT system is not straightforward. Here the design steps are shown with the help of a numerical example. The case chosen for illustration is based on a circuit for which the distance between the two coils is $100 \mathrm{~mm}$; however, this could be done for any distance, and the only condition that has to be fulfilled is that the source impedance has to be smaller than the input impedance. In the case of a $100 \mathrm{~mm}$ distance between the coils the input impedance is $108.25 \Omega$, whereas the source impedance is $0.1 \Omega$. In this arrangement the input power is about $46 \mathrm{~W}$ and the efficiency of the wireless power transfer is about $92 \%$.

The design process starts with calculating the value of inductance $L$ used to resonate the two capacitors in the tapped capacitance network. The value of $L$ can be calculated as long as the loaded quality factor $\left(Q_{L}\right)$ for the magnetic coupled resonators from the WPT system is known. For a load of $11.75 \Omega$ the $Q_{L}$ of this system is 10.84 . The inductance $L$ has to have the same loaded quality factor to preserve the bandwidth of the coupled resonators. Using this information one can write the first design equation as

$$
Q_{L}=1 /\left(\omega_{0} L\left(G_{L}+G_{P}\right)\right)
$$

where $G_{L}$ is the conductance associated with the load, which in this case is $1 / Z_{\text {in }}$, noting that at resonance the $Z_{\text {in }}$ is purely real, and $G_{P}$ is the conductance associated with the inductance $L$. Assuming that the unloaded quality factor of the inductor $L$ is 120 , which is a typical value for inductors at this frequency, the second design equation can be written

$$
Q_{U}=1 /\left(\omega_{0} L G_{P}\right)
$$

Using (10) and (11) the value of the inductance $L$ can be calculated. Once the inductance is known the total capacitance $\left(C_{A} C_{B}\right) /\left(C_{A}+C_{B}\right)$ can be found from the third design equation

$$
\left(C_{A} C_{B}\right) /\left(C_{A}+C_{B}\right)=1 /\left(\omega_{0}^{2} L\right)
$$

Finally, the values of the two capacitors $C_{A}$ and $C_{B}$ can be calculated using (12) and (13).

$$
\left(1+C_{B} / C_{A}\right)=\sqrt{Z_{\text {in }} / R_{S}}
$$

The above methodology was applied to the case which had the two coils separated by a distance of $100 \mathrm{~mm}$. In this case, as explained earlier, the input impedance of the WPT system is $108.25 \Omega$. Using (10) and (11) the inductance in this particular case was calculated to be $15 \mu \mathrm{H}$. From (12) the total capacitance at $96.5 \mathrm{kHz}$ is calculated to be $180 \mathrm{nF}$. Finally, using (13) in conjunction with (12), the two values of the capacitance are computed: $C_{B}$ is $5.9 \mu \mathrm{F}$ and $C_{A}$ is 185.64 $\mathrm{nF}$.

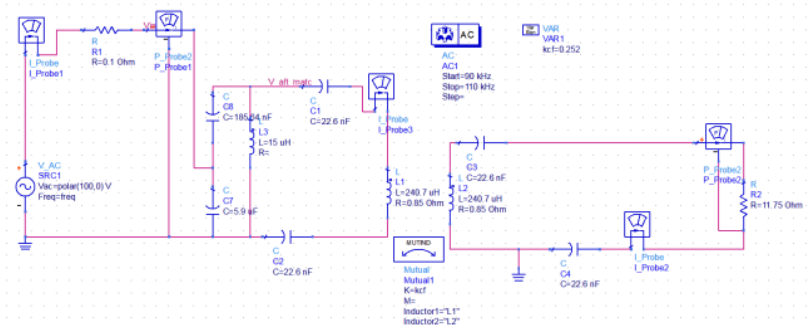

Fig. 7. WPT Equivalent circuit model with matching tapped capacitance impedance transformer introduced between the source and the primary coil of the WPT system.

The tapped capacitance network, together with the equivalent circuit of the WPT, was modelled using a circuit simulator (Fig. 7). The tapped capacitance impedance transformer reduces the impedance seen from the source into the WPT system to a low value of $0.28 \Omega$ (Fig. 8.a); as a result the input power available now is $4,598 \mathrm{~W}$ (Fig. 8.b) as compared with the input power of $46 \mathrm{~W}$ available before the introduction of the matching stage. The efficiency of the wireless transfer is unchanged as the quality factor of the magnetically coupled resonators was not affected by the impedance transformation, therefore the power transferred to the load is now 4,254 W (Fig. 8.b).

It is clear from the above simulation results that the tapped capacitance impedance transformer network has a beneficial effect over the amount of power that can be transferred through the WPT system. However, the transformer network has also increased the voltage levels at the input and output of the WPT resonating coils and the practical implication of this 
high voltage has been not been yet fully considered. Further experimental lab work will be necessary to verify the simulation results presented here and to fully understand the practical design implication of using such a tapped capacitance impedance transformer network.
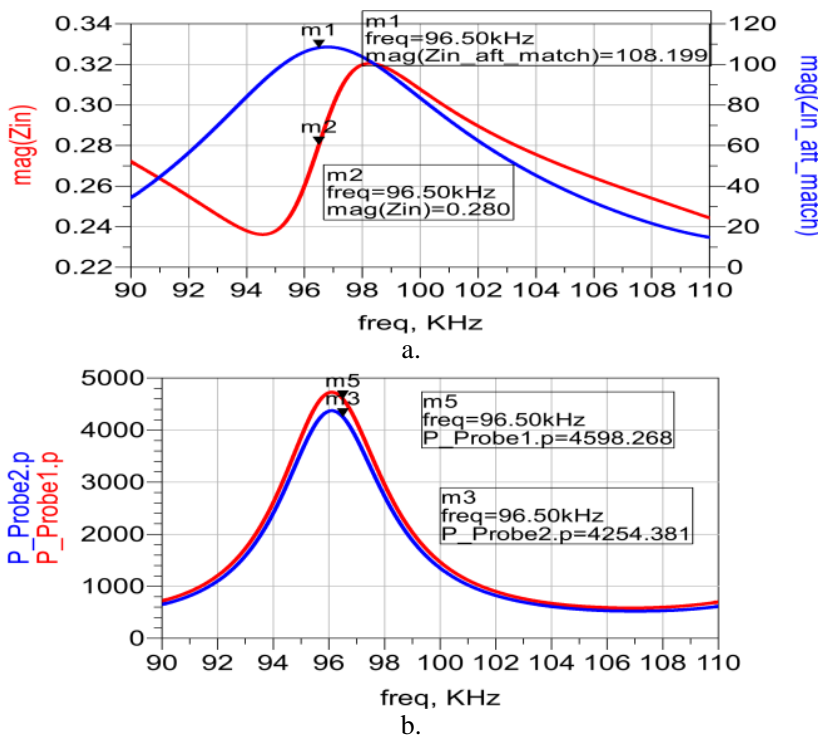

Fig 8. a. Real part of $Z_{\text {in }}$ and impedance after the matching stage when load impedance is $11.75 \Omega$ and distance between coils is $100 \mathrm{~mm}$. b. Power in and power delivered to the load when the tapped capacitance impedance transformer is used.

\section{EFFECT OF MASSIVE CONDUCTING SHIELDS}

Another aspect that has to be considered when WPT systems are used in higher power applications, such as electrical vehicles, is the proximity effect due to other large conductive bodies. In our previous work [10] we have used a full wave 3D simulation to extract the equivalent circuit of the magnetically coupled resonator. The same methodology was used here to extract an equivalent circuit model that includes the effects of a conductive body in the proximity of the WPT system.

A 3D model of one coil with a $200 \times 200 \mathrm{~mm} 5 \mathrm{~mm}$ thick aluminium plate above the coil at a variable distance was constructed and solved in frequency domain. The distance between the coil and the aluminium plate was varied between $20 \mathrm{~mm}$ and $100 \mathrm{~mm}$. The excitation used for this simulation was a current source with an impedance of $50 \Omega$. The source produces a $1 \mathrm{~W}$ input power, which enables the calculation of scattering $(S)$ parameters. The $S 11$ parameter was obtained for a frequency sweep from $50 \mathrm{~Hz}$ up to $1 \mathrm{MHz}$. The results for four different distances are presented in Fig. 9. It is clear that the resonant frequency of the coil with the metal plate in its vicinity is different from the resonant frequency of a single resonant coil with two series capacitors. It is also apparent that the distance between the coil and the metal slab influences the resonant frequency. In an attempt to explain the effects of the metal slab on the WPT system, an equivalent circuit for one resonant coil with the metal plate in

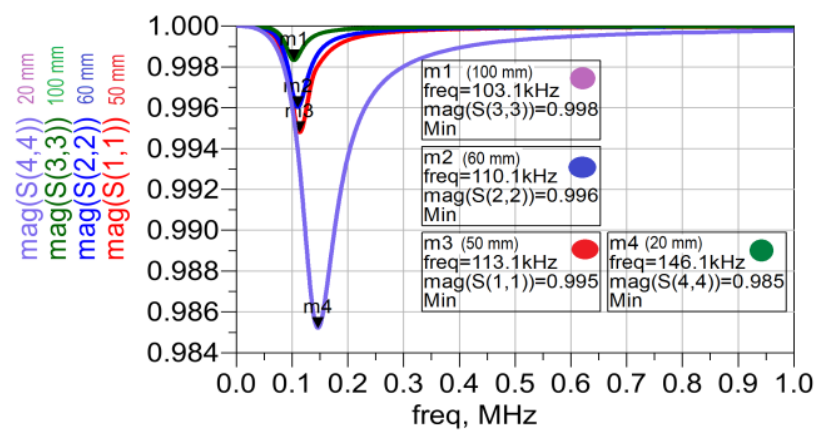

Fig 9. Magnitude of S11 for four different distances between coil and conducting plate - distances were $20,50,60$ and $100 \mathrm{~mm}$ respectively.

its vicinity was extracted.

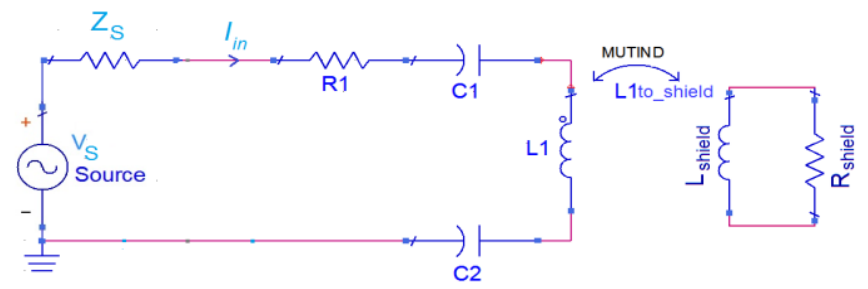

Fig. 10. Equivalent circuit model for one coil with a conductive shield plate.

It was expected, and indeed shown in $3 \mathrm{D}$ simulation, that there would be induced eddy currents in the conducting plate; this current will produce its own magnetic field and it will be dissipated through ohmic losses. To model this situation the equivalent circuit in Fig. 10 is proposed, where the conductive plate is represented by an inductance in series with a resistance which is magnetically coupled to the coil.

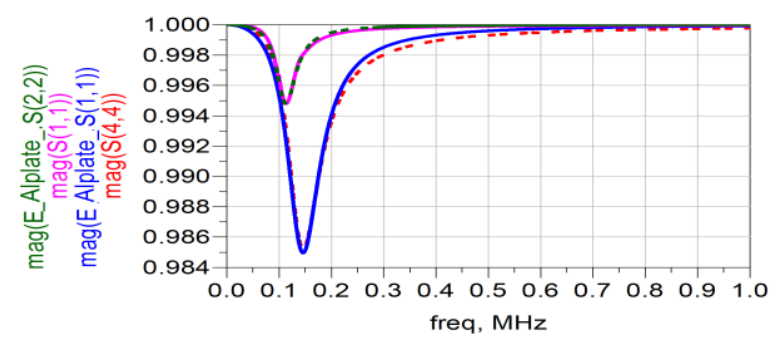

Fig. 11. Comparison between equivalent circuit results (dotted lines) and full wave $3 \mathrm{D}$ model results (full line) for the one coil with a conductive shield$50 \mathrm{~mm}$ and $100 \mathrm{~mm}$ case.

The circuit in Fig. 10 was solved in frequency domain and the results in terms of $S$ parameters were used to match the results obtained from the $3 \mathrm{D}$ simulation models. Using this approach the inductance of the shield, the coupling coefficient to the coil and the resistance of the plate were found. The comparison between the full wave 3D model results and the results obtained using the equivalent circuit in Fig. 10 are presented in Fig. 11. A very good agreement has been achieved.

Analysing the input impedance of this circuit reveals that the effective inductance drops while the real part of the input impedance increases (14). The drop in inductance results in a shift of the resonance towards higher frequency, while the increase in the real component of the impedance leads to a 
drop in the quality factor. It is also clear that reducing the distance between the coil and the conductive plate will amplify the effects mentioned above.

$$
\begin{aligned}
& Z_{\text {in }}(\omega)=\left(j \omega L_{1}+1 /\left(j \omega C_{1}\right)+1 /\left(j \omega C_{2}\right)+R_{1}\right)+ \\
& \left(\omega^{2} \cdot L_{1 \text { toshiled }}^{2}\right) /\left(j \omega L_{\text {shield }}+R_{\text {shield }}\right)
\end{aligned}
$$

The circuit model of a full WPT system, which accounts for the effects of a conductive plate, was built and validated using a similar methodology as described earlier and is

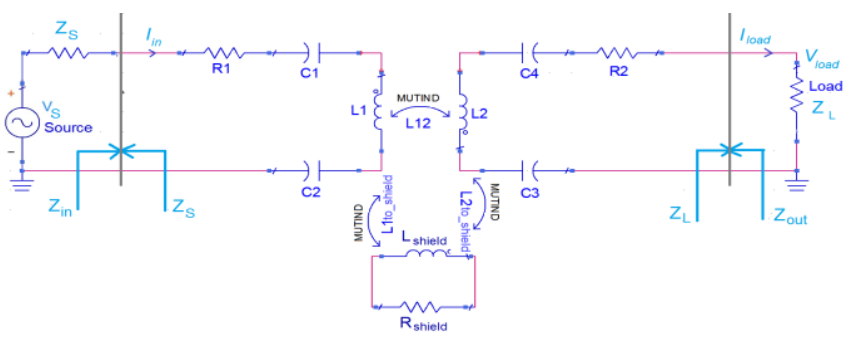

Fig. 12. Equivalent circuit model of the WPT system with a conductive shield plate.

presented in Fig. 12. The model is extracted for the case where the conductive plate is near the secondary coil. There is a need to reduce the magnetic field directly above the secondary coil, hence this model tries to simulate this situation. The 3D full wave results were used as validation criteria. As before, a very good agreement between the results from the equivalent circuit model and the full wave 3D model results have been observed.

As in the case of a single coil, a shift towards a higher frequency of the resonant frequency has been observed when the conductive plate is introduced near the resonant coils. There is also a drop in the efficiency of the system, which

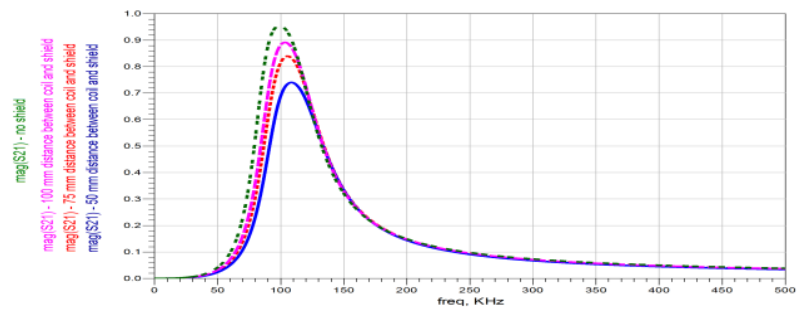

Fig. 13. Variation of the S21 magnitude for different distances between the secondary coil and the shield.

may be directly linked with the drop in the quality factor of the resonators. As in the single coil case, the closer the plate is to the coils assembly the higher the shift in the resonant frequency (Fig. 13).

\section{CONCLUSIONS AND FUTURE WORK}

The wireless transfer systems based on magnetically coupled resonators can offer benefits and advantages for applications such as electric vehicle charging; however, there are several design challenges that need to be addressed before such systems will become widely accepted. From the equivalent circuit model it is clear that maximizing the quality factor as well as minimizing the reflections between the power source and the loaded WPT system is very important in achieving high efficiency wireless power transfers.

The work in this paper has proposed a matching solution between the power source and the loaded WPT system based on a tapped capacitance impedance transformer. The results obtained from circuit simulation demonstrate that such an approach will be very beneficial to WPT systems. With the correct matching network in place, theoretically very large amounts of power could be transferred through the WPT system at very high efficiencies. The simulation results are very encouraging and a practical implementation of the proposed matching network is planned.

Another practical aspect of the WPT system has been also discussed in this paper, namely the effect of massive conducting structures on the efficiency and behaviour of the WPT systems. An equivalent circuit model, which includes the effect of such conductive structures, has been proposed and supported by 3D full wave simulations. Going further it is expected to validate these results not only through simulations but also with experimental results. It is also intended to develop an impedance matching approach that could reduce the detuning effects of the massive conducting structure found in the vicinity of such WPT systems.

\section{REFERENCES}

[1] Howard R. Ross, "Roadway for supplying power to vehicles and method of using the same," U.S. Patent no. 4007 817, Feb. 15, 1977.

[2] S. Ahn, J. Pak, T. Song, H. Lee, J. Byun, D. Kang, C. Choi, E. Kim, J. Ryu, M. Kim, Y. Cha, Y. Chun, C. Rim, J. Yim, D. Cho, and J. Kim, "Low frequency electromagnetic field reduction techniques for the online electric vehicle (OLEV)", Proc. of 2010 IEEE International Symposium on Electromagnetic Compatibility, Jul. 2010.

[3] Kurs, A. Karalis, R. Moffatt, J. D. Joannopoulos, P. Fisher and M. Soljacic, "WPT via strong coupled magnetic resonances," Science Express, vol. 317, No. 5834, pp. 83-86, June 2007.

[4] Sample P. Alanson, David A. Meyers and Joshua. A. Smith, "Anlysis, experimental results and range adaptation of magnetically coupled resonators for wireless power transfer," IEEE Trans. On Industrial Electronics, vol. 58, No. 2, pp. 544-554, February 2011.

[5] W. Q. Niu, W. Gu, J. X. Chu and A. D. Shen, "Coupled-mode analysis of frequency splitting phenomena in CPT systems," ELECTRONICS LETTERS, vol. 48, No. 12, 7th June 2012.

[6] Mhedi Kiani, and Maysam Ghovanloo, "The circuit theory behind coupled-mode magnetic resonance-based wireless power transmission," IEEE Trans. On Circuits and Systems, vol. 59, No. 9, pp. 2065-2074, September 2012

[7] Erin M. Thomas, Jason D. Heebl, Carl Pfeiffer and Anthony Grbic, "A power link study of wireless non-radiative power transfer system using resonant shielded loops," IEEE Trans. On Circuits and Systems, vol. 59, No. 9, pp. 2125-2136, September 2012.

[8] Jesus Sallan, Juan L. Villa, Andres Llombart, and Jose Fco. Sanz "Optimal design of ICPT systems applied to electrical vehicle battery charge," IEEE Trans. On Industrial Electronics, vol. 56, No. 6, pp. 2140-2149, June 2009.

[9] Linhui Chen, Shou Kiu, Yong Chou Zhou, and Tie Jun Cui, “An optimizable circuit structure for high-efficiency wireless power transfer," IEEE Trans. On Industrial Electronics, vol. 60, No. 1, pp. 339-349, January 2013.

[10] Mihai D. Rotaru, Robin Tanzania, Raed Ayoob, Tan Yen Kheng and Jan K. Sykulski, "Numerical and experimental study of the effects of load and distance variation on wireless power transfer systems using magnetically coupled resonators", CEM 2014, London, March 2014.

[11] Kenneth C. Clarke and Donald T. Hess, "Communication Circuits: Analysis and Design”, Krieger Publishing Company, 1994. 